\title{
Enriched Media-Experience of Sport Events
}

\author{
Josef Hallberg, Sara Svensson, Ake Östmark, Per Lindgren, Kåre Synnes, Jerker Delsing \\ Luleå University of Technology \\ Department of Computer Science \& Electrical Engineering \\ SE-971 87 Luleå, Sweden \\ \{Josef.Hallberg, Sara.Svensson, Ake.Ostmark, Per.Lindgren, Kare.Synnes, Jerker.Delsing $\} @ 1 t u . s e$
}

\begin{abstract}
This paper describes a system where Internet-enabled sensor technology was integrated into a context-aware platform to give viewers of sport events an enriched media experience. The system was developed as a proof of concept and was evaluated during real-life use at the Vasaloppet crosscountry ski event. Using Bluetooth wireless ad-hoc networking and GPRS technology, sensor data was transmitted from contestants to the context-aware platform Alipes, which in turn presented the sport event viewer with a personalized, context-aware view. In this paper we discuss the system architecture and integration of components. The system was evaluated both from technical and user perspectives, where the evaluation results confirm our approach to be technically feasible and that the system provide an enriched media-experience for the majority of viewers.
\end{abstract}

\section{Introduction}

During the last decade, the usefulness of contextawareness has been shown in a number of scenarios, e.g. tourist guides [1,7], reminder systems [15, 25] and office applications [31, 33]. One area which has received less attention is sport events, in which information about contestants often is very sparse. The information is typically limited to name, age, country, and, if applicable, place and elapsed time. By equipping the competitors with sensors, additional information, such as pulse and location, can be retrieved. This information could then be provided to the viewer to achieve an enriched experience. The information could be provided both as it is and in some way refined, for example as comparisons between contestants' pulse during the race. With the help of location information from participants, an application could also enable the viewers to follow the participant of their choice. Hence, they could follow their favourite athlete, a friend, or a relative, rather than only following the contestants who are in the lead, which often is the case in traditional broadcasting media.

One way of accomplishing this would be to incorporate the new information and possibilities of choice into a regular or interactive television broadcast. A more easily deployable way may be to implement it as a web application, in which viewers can access the content of their choice. Would any viewers be interested in these new possibilities? Could a working solution be built with the Internet enabled sensors and the context-aware platform developed in our research? To get an indication of public interest, we built a web application as a proof of concept, where the location, pulse, and speed of cross-country skiers could be followed. This would also allow us to study how well the sensors perform under extreme working conditions as well as study scalability issues of the context-aware platform in real life operation.

We deployed and tested a prototype system during the world's largest skiing event, the Vasaloppet week, which is held annually in Sweden during one week in the beginning of March [30]. The main event of the Vasaloppet week is the 90 kilometres contest, Vasaloppet, which is complemented with a whole range of other cross-country skiing events. One of these events is the open track non-competitive event, where the participants may start at any time within a given time frame to conclude the 90 kilometres at their leisure. Our application was tested both during Vasaloppet and during the open track event. Three professors from Luleå University of Technology, one of them shown in figure 1 practicing cross-country skiing, participated in the testing by taking part in the Vasaloppet contest and the open track event equipped with sensors.

Related work is described in the following section. Section 3 gives a detailed view of how the system is implemented and of how it operates. Section 4 presents the results from the tests during the Vasaloppet week and section 5 discusses conclusions while section 6 describes visions and future work. 


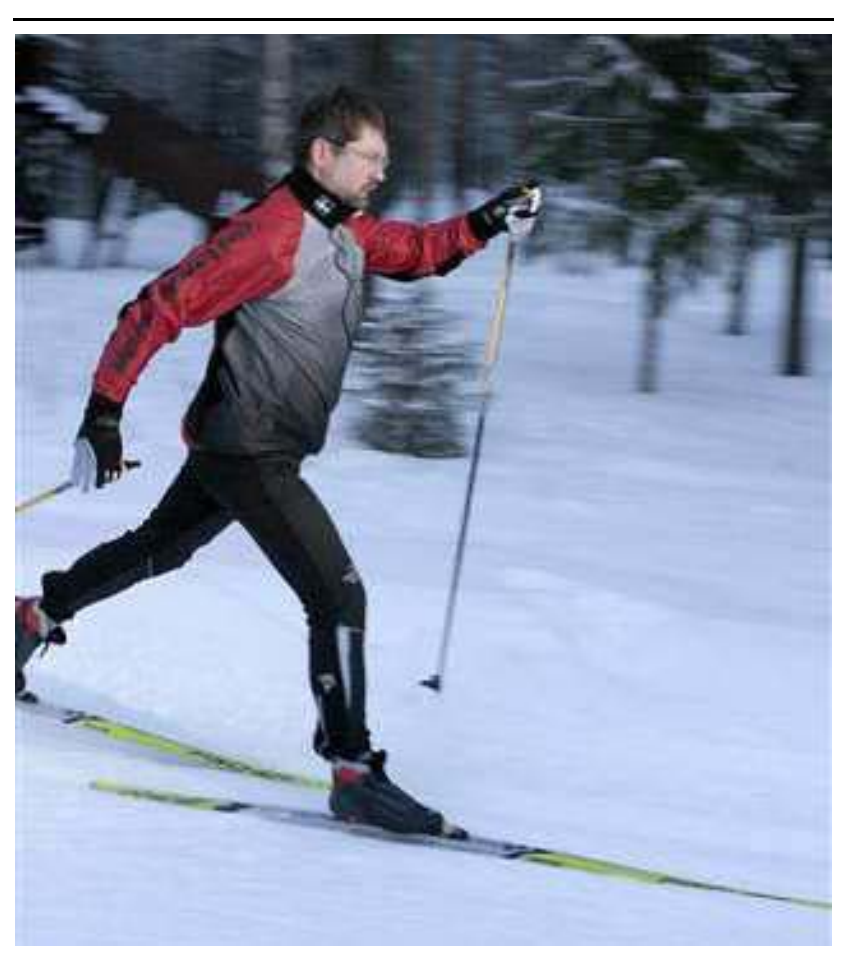
Figure 1. Cross-country skiing (photo: Per
Pettersson)

\section{Related work}

Previous research has been conducted on trying to enrich users' interest, engagement, and experience of media in different ways [6, 8, 21, 22]. However, few have focused on sport events and the approach to give the viewer an alternative view or option of whom to follow in these events has not been utilized.

Some work close to our application is the Arena project [3], where hockey players were equipped with sensors to enrich viewers' experience. Some of the big differences compared to our work are that the Arena project is focused on team sports and that the viewers could see the whole game even without the equipment. In cross-country skiing you do not have the possibility to completely follow a whole race neither by being there in person nor by watching television broadcast. One approach to enable viewers to more closely follow athletes during these types of sport events has been the use of RFID tags. This makes it possible to know when athletes pass certain points and average status between two points but it doesn't allow a viewer to know the status of an athlete at any given point during the race.

The system is built on a context-aware platform, Alipes [20] and wireless Internet enabled sensor nodes [36] developed at Luleå University of Technology. Other research conducted on context-aware platforms includes the the work by Dey et al. on the Context Toolkit [9] and the work by Romàn et al. on Gaia [26]. While both of these platforms could have been used in this project, they are not originally designed for sharing personal context with others, which is the case with the Alipes platform.

Quite a few research prototypes of wireless senor nodes have been designed and manufactured e.g. the Mica mote $[12,13], \mu$ AMPS [19], the MANTIS Nymph [2], and the GNOMES node [34]. Many of those devices uses commercial off-the-shelf (COTS) components. Especially the GNOMES node looks promising to be used in a project like this due to the communication capabilities, but the system software needed to achieve interoperability with existing systems seems only to be partially implemented.

\section{System}

Overall, the system is intended to give the viewer an enriched experience by providing additional context to the sport event. An overview of the system is illustrated in figure 2. The viewer, A, is presented with a Java applet showing contestant information, see subsection 3.1. The applet gains its information from the context-aware platform, B, described in subsection 3.2. Data is received via a wireless network, C, from the sensor nodes in the ad-hoc network, $\mathrm{D}$, as described in subsection 3.3.



Figure 2. Overview of the system

\subsection{Viewer applet}

The purpose of the applet is to show the advancement and status information of contestants during Vasaloppet. The monitored skiers were equipped with sensors measuring altitude, position, pulse, and speed. During the race 
the collected values are sent from the sensors via General Packet Radio Services (GPRS) to the database of a contextaware platform.

The applet extracts the most recent data from the platform's database at regular time intervals and displays them. The skiers' locations are drawn on a map which the user is able to zoom and pan. The map can also be centred on one or all of the skiers' locations by pressing one of the top buttons shown in figure 3 below. Pulse and altitude are drawn in diagrams related to the distance each skier has covered. Each skier has his own pair of diagrams, which scroll horizontally according to the current distance covered. The name of the skier is displayed at the top right corner of his diagram pair. Digital counters are placed at the right side of the diagrams. The digital counter displays the skier's speed in kilometres per hour in the altitude diagram and the current value of the skier's pulse in the pulse diagram. The applet is depicted in figure 3 below.

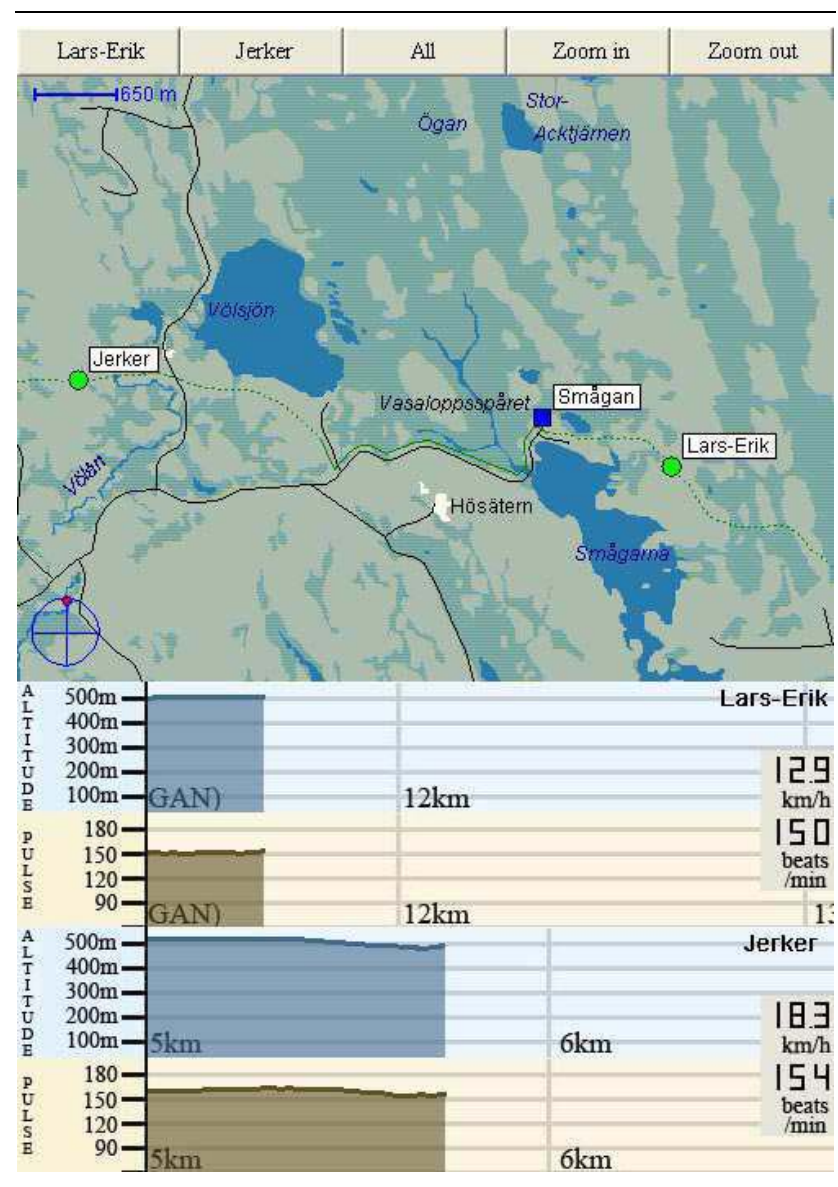

Figure 3. The Vasaloppet applet

\subsection{Context-aware platform}

The function of a context-aware platform is to encapsulate management of sensors and other data providers [9], handle information fusion $[11,24,35]$, and deal with privacy issues [14]. Alipes [20], the platform used in this project, is one such platform. It was originally developed for location aware applications but has been extended with support for other types of contexts; altitude, pulse, speed, and distance, for the purpose of conducting this test.

The Alipes architecture includes a SQL database and can function as a proxy. Sensor-data is sent via the mobile device and is stored in the database. A client which is monitoring the progress of the race contacts the database and is given the latest altitude, distance from start, position, pulse, and speed. This means that the different contexts might not be from the exact same time. In the event of one sensor not reporting any new data, the latest data will remain unchanged except for the position, which will be estimated based on latest speed and knowledge about the Vasaloppet track. In this way it is possible to reduce the negative effects of temporary network or sensor failures. Assuming that extrapolated data has value for the viewer, no limit was applied to how old data could be until it was considered irrelevant to the current situation.

Although privacy is an important aspect in context-aware systems it was not utilized in this project, mainly because the whole point of the project was to distribute the information to the public. There is however a rule-based system for privacy in Alipes [28] that could be used if there was a need.

\subsection{Sensors}

A number of sensors exist to monitor heart rate. Of course, a cross-country skier would be impeded by wearing sensors like finger clip sensors. During the ski competition event, a belt worn around the chest [23] was used. Signals from the chest belt are transmitted wirelessly to a receiving unit, in the same way as in equipments such as treadmills and exercise bikes. For collecting the position, time and velocity of the ski-runners, a low power GPS module [16] was used.

3.3.1. Generic sensor hardware platform. Both the receiving unit for the heart rate monitoring and the GPS module was interfaced by a small mobile sensor node developed at Luleå University of Technology. The sensor node is a hardware device having a 16-bit microcontroller [18] used for processing of received signals and wireless communication. In general, wireless communication is the major power consumer in mobile devices [32]. For low power wireless communication, the node has a Mitsumi Bluetooth module [4]. The components, microcontroller, Bluetooth module, and batteries are mounted in a box $(11 \times 6.5 \times 2 \mathrm{~cm})$ as 
illustrated in figure 4 below. The weight of the GPS sensorbox and heart rate sensor-box is $145 \mathrm{~g}$ and $130 \mathrm{~g}$ respectively.
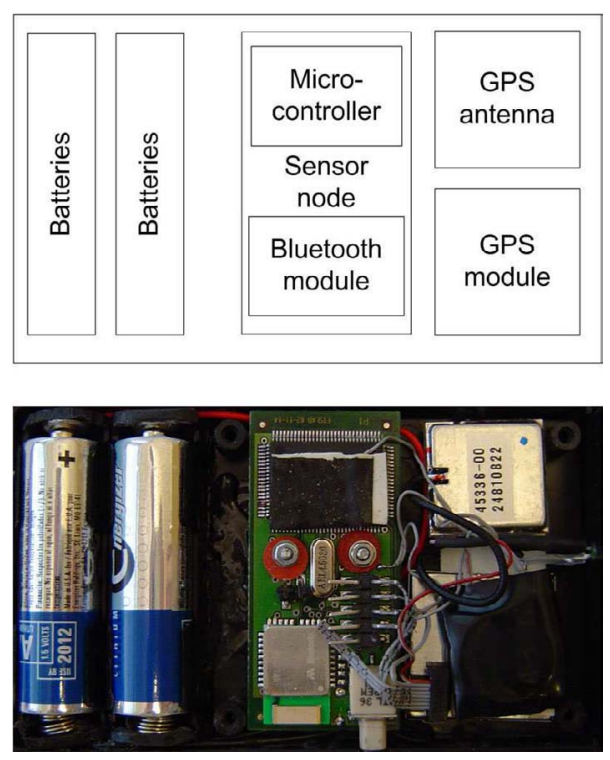

Figure 4. Hardware components

3.3.2. Sensor node software. Each of the competitors had a Bluetooth/GPRS-enabled mobile phone, which was used as an access point. GPRS facilitates almost instant connections where data can be sent (or received) immediately as the need arises, of course under subject to radio coverage and GPRS channel availability. Utilizing GPRS and the Internet Protocol (IP) for communication has numerous advantages over developing proprietary protocols, e.g., compatibility, flexibility, and ease of maintenance. To be able to run the TCP/IP stack on a sensor node with limited resources, a stack with focus on low resource utilization [10] has been used.

For communication between the mobile phone and the sensors (i.e. sensor nodes), a Bluetooth stack was developed [17] extending the TCP/IP stack with Bluetooth access capabilities. The Bluetooth standard defines a set of profiles for communication. Today, a mobile phone generally implements the capabilities of the Dial-Up Networking [5] profile by acting as a wireless modem. However, during operation, only one Bluetooth device may use the dial-up or GPRS services at a time. Since the minimum configuration during the Vasaloppet competition was to have two sensor-nodes worn by the participants, the LAN Access Point (LAP) [5] profile was implemented to give Internet connectivity for the second device. This architecture is not limited to having only two nodes, but can be extended to several nodes due to the LAP service implementation. The purpose of having one sensor-node attached to each sensor is to minimize the need for cables and increase flexibility. Without wires, a selection of sensor-nodes interfacing different types of sensors can be used to be worn or carried by skiers.

3.3.3. Sensor node communication and data acquisition. When started, the sensor node initiates an inquiry to find other Bluetooth devices in the close proximity that provides Internet access. When a device is found and the connection is established, the device acts as a LAP providing other sensor nodes with the possibility to get connected. At any time, the nodes may lose their connection due to a number of reasons. The mobile phone might be out of range of a base station, data sent over GPRS might be dropped due to the policy of prioritizing voice. For some reason, it is also possible that the competitors may leave the mobile phone too far away (approximately 10 meters) from the sensor nodes. This implies that the sensor node must be able to form a spontaneous, or ad-hoc network, able to re-establish connection. When the device has established connectivity, either by using the mobile phone or the other sensor node, readings from the attached sensor is performed and data is transmitted over the public network. To reduce the sending rate, collected data from the sensor can be processed before sending.

\section{Results}

The three professors, henceforth called Professor A, B, and $\mathrm{C}$, participated in either one or both of the open track event and the Vasaloppet race. During the events, four sensor nodes were used. Professor A participating only in the open track event, and professor C, participating only in Vasaloppet, used the same pair of sensor nodes. Professor $\mathrm{B}$ used the other pair and participated in both events. The results acquired from the races have been divided into four categories: Viewer evaluations, Skier evaluations, Contextaware platform, and Sensors and communication. The results for these categories are presented in the following subsections.

\subsection{Viewer evaluations}

A questionnaire was sent out on Testbed Botnia [29], which is one of Sweden's first and largest open testbeds for mobile services. Anyone who has a mobile phone and accepts the terms of participation is allowed to join the testbed. The testbed currently has more than 5800 members and out of these 89 members tried our applet and answered our questionnaire. The participants of the survey ranged from age 12 to age 57 with an average age of 31 . The participants were asked about how long they had visited the webpage and about their interest in sports and new technology as well as if they felt that the added information enhanced their experience of the sport event. 52 of the 
participants visited the webpage for no more than $15 \mathrm{~min}$ utes, while the remaining visited for a longer period of time, some for more than an hour. Most of the participants (all but 3 ) were either interested or very interested in sports $(2 \%)$, in new technology $(28 \%)$, or in both $(66 \%)$. Over all, $84 \%$ thought that the added information enriched their experience. All of these participants belonged to the group that were interested in sports and/or new technology.

According to the survey, being able to see the locations of the skiers on a map was especially helpful. Although people liked the idea and the information in general, they saw a problem with some of the information, especially pulse data, not updating at all times and the maps being slow to load. The full survey with answers can be viewed in [27].

\subsection{Skier evaluations}

From the skiers point of view, the sensors were simple to wear and handle, heart rate sensor attachment was a simple strap belt around the chest. The two sensor electronics boxes had only an on/off operation switch each and were positioned in suitable pockets of the skiing dress. The whole system was started by turning on the mobile phone and switching on the sensors, after which the skier could simply forget about them.

The batteries in the sensors had a lifespan of a few days. Thus, the limiting factor was the mobile phone, where batteries had roughly one day of operation time. The data obtained was of great interest to the athletes because data analyses after the race gave clear information about the athlete's performace. This can be used to improve training for the next race. The live Internet sensor data from the athletes actually triggered people to call up one of the athletes during the race and comment on position, speed and heart rate. One of the professors believed that even professional skiers might agree to wear this equipment, especially if other contestants also wore it.

\subsection{Context-aware platform performance}

During the day of the Vasaloppet race there were 2100 unique visitors to the public website with the applet. The average load to the database was about 120 simultaneous connections. The amount of visitors caused some problems for the map-server that had to serve at least 120 people with a map-image. In the beginning of the test there was also a problem with the user limit to the database which was set to 100 simultaneous users. However, this limit was removed after less than two hours into the race.

\subsection{Sensors and communication}

Professor A. This professor only participated in the open track event. Below in figure 5, a graph of the heart rate values from the professor, represented as beats per minute (bpm) is shown for his race.



Figure 5 . Heart rate of professor A through-
out the open track event

During the open track event, more than 8000 heart rate samples were collected and transmitted from the sensor node. The final time of this skier was approximately 12 hours and during that time, the sensor nodes were operational for more than $97 \%$ of the time.

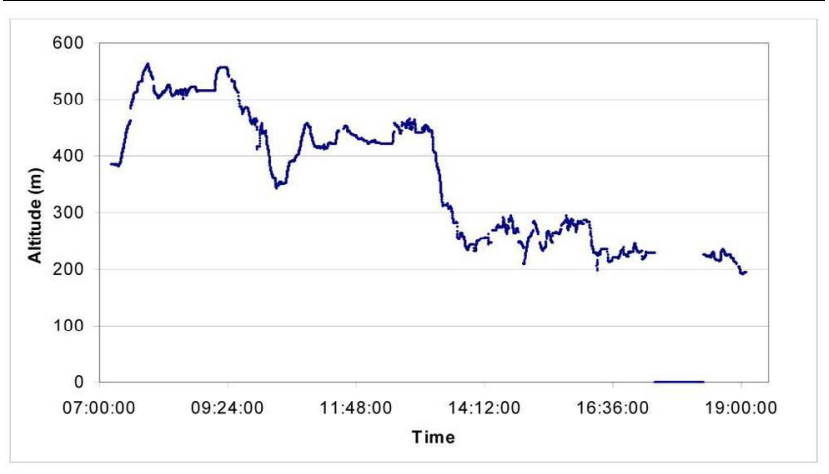
Figure 6. Altitude measured for Professor A
throughout the open track event

Using the GPS module makes it possible to track e.g. the position and velocity of the ski-runners. In figure 6 above, the altitude is shown for the 12 hours race. In the above graphs there are flat lines at approximately 5-6 P.M. During that time, the skier was indoor resting for the final 10 
$\mathrm{km}$ of the race. The equipment was placed on the floor and hence, neither position nor heart rate could be determined, but still, the sensors were operational. Moreover, during the final $10 \mathrm{~km}$ the batteries of the mobile phone were used up completely. Fortunately, an extra phone was available for the sensors to connect to and as a result, the cross-country skier could be monitored until the end of the race.

Professor B. The second professor participated in both of the races, having a final time of approximately 6 hours in both of the races. For the open track event, his position and speed could be observed for $90 \%$ of the event, but the sensor node attached to the heart rate receiver malfunctioned; only transmitting data for the last $10 \%$ of his race. One week later during the main Vasaloppet event, the equipment continued to be unstable but nevertheless, his position and heart rate could be monitored for 2 and 3 hours respectively of the competition.

Professor C. In figure 7 below, the position of the third professor participating in Vasaloppet is plotted on a map during the first $13 \mathrm{~km}$ of the competition.

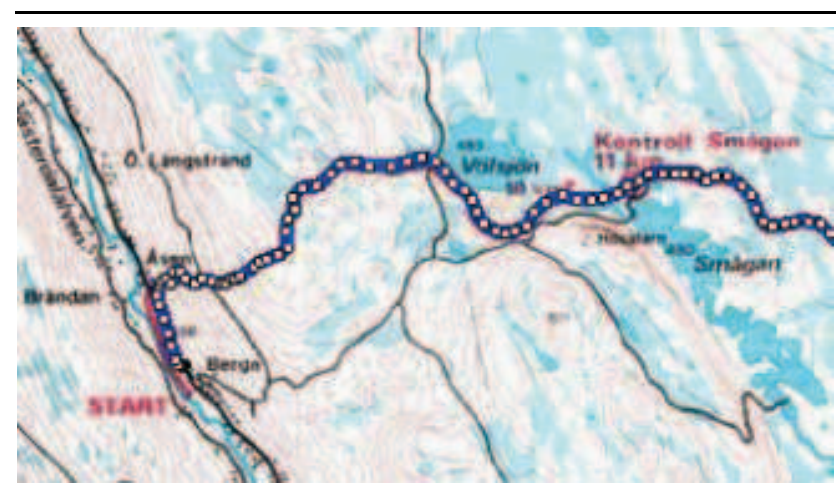

Figure 7. Transmitted position during the first $13 \mathrm{~km}$ of Vasaloppet for Professor C

The sensor node attached to GPS module and senor node attached to the heart rate receiving unit transmitted data for $69 \%$ and $76 \%$, respectively of the time during the $90 \mathrm{~km}$ race. The on-line operational time is shown in figure 8 below.

In figure 8, gaps along the time axis represents missing data from the senor nodes. The professor had a final race time of almost 10 hours, and in particular for the GPS node, we find 3 large gaps of approximately 30 minutes each where no data could be sent. The bandwidth requirement for sending data over the wireless link was low (for GPS, only 80 bytes of payload every fifth second) but nevertheless, the radio channel is scarce resource.

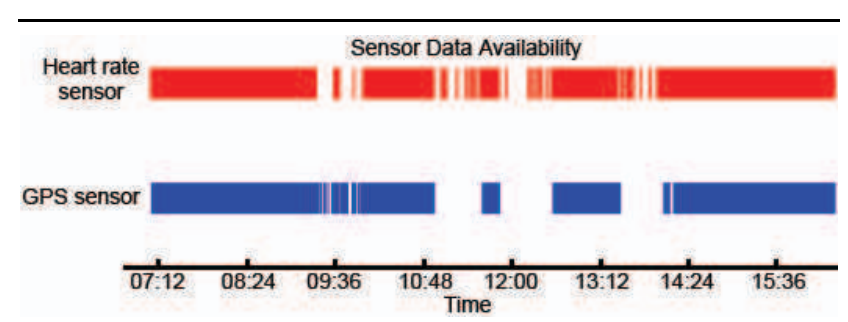

Figure 8. Operational time for mobile units during Vasaloppet for Professor C

\section{Conclusions}

The results from the survey indicate that the added information did indeed enrich viewers' experience of the event, at least for those viewers who have interest in sports or technology. A problem that many from the survey noticed was that the pulse sensors didn't work very well. This problem is most likely due to the active movement of the skier causing the sensor to slide out of position and thus making it unable to read the pulse. However, this is just one possible cause, other causes could be communication problems between the sensor and the mobile-phone or battery shortage. One possible way to minimize this problem could be to give sonic feedback whenever any problem occurs (communication or sensor problems).

One bottleneck in the system was the map server as it had to provide a new map image for every user whenever they changed view or the application itself requested a new and updated map image. The map is generated from centre and edge coordinates, then it is converted into the requested image format. A system where a limited number of different views are offered, thus limiting the generation and conversion process, would greatly improve the system in terms of scalability. The different views could be cached on a server to further improve the system performance.

The largest amount of data was received during the open track event. Achieving $100 \%$ uptime of the sensor nodes is very hard with the limited resources on the nodes. The lower operational time of the mobile units when used in Vasaloppet is most likely due to insufficient GPRS-resources available due to the increased amount of spectators, participants and media coverage. This means that the limited number of available GPRS time-slots will make it hard to deploy the system for many athletes. Unfortunately, professor B participating in both of the races had the set of sensors providing the lowest operational time. The cause is still unresolved, however the sensor nodes proved their robustness by reconnecting when possible. That is, even though not having enough resources available for some time; the sensor nodes were never user-operated and were able to reconnect 
when resources (GPRS) were available. Ongoing research is targeted to improve robustness and reducing power consumption of the sensor nodes. We also expect future cellular phones to provide increased operational time.

All in all, according to the survey, sensor results, and the interest shown for the concept by the large amount of visitors, we consider this to be a successful first trial in the area of enriched sport events. For future trials we have identified the bottlenecks needed to be addressed.

\section{Visions and future work}

One of the future visions includes the possibility to view any contest participant with complete information at any time during the contest. With several cameras positioned along the track and by utilizing location-awareness a viewer could select to automatically switch to a camera currently filming a certain contestant. This kind of system could be developed for both Internet and television by using new technology in digital television.

A project, under the name of LIVEStat, has been created in order to further develop the concept presented in this paper. The project strives to achieve a solution to present the concept not only on the Internet but also together with live television broadcasting. Using this media channel has proven to offer a way to further increase the versatility of the graphics presented and also to cover more conceptual areas.

The different contexts described in this paper has proven to be interesting, however there is yet another context that could be of interest: the time displacement. In a sport event where contestants have different starting times, a comparison of physical location of contestants at a certain progressed time can be displayed on a map. The benefit of time displacement is well displayed in orienteering as seen in figure 9 where the different approaches to the control point are shown with regards taken to each contestant's start time.

\section{Acknowledgements}

This work was sponsored by Vinnova, the Centre for Distance-spanning Health-care $(\mathrm{CDH})$ and by European structure funds (mål1) through the Centre for Distancespanning Technology (CDT). The authors would like to thank Cartesia for providing maps, Morastrand for making race data available and TeliaSonera for allocation of a GPRS data slot. The authors would also like to thank Jonas Thor for his work with the sensors, Ronny Pekkari for his PR work, the LIVEStat people for help with the visions and future work section, and Mikael Drugge for his helpful comments on this paper. Finally, the authors would like to acknowledge the three professors for participation and carrying the equipment throughout the Vasaloppet event.

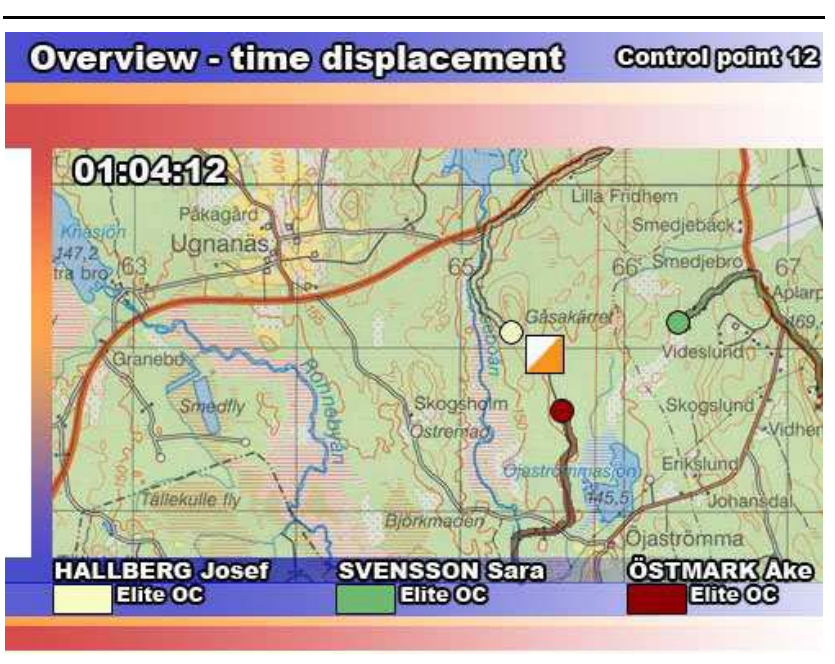

Figure 9. The time displacement map in an orienteering scenario

\section{References}

[1] G. D. Abowd, C. G. Atkeson, J. Hong, S. Long, R. Kooper, and M. Pinkerton. Cyberguide: a mobile context-aware tour guide. Wireless Networks, 3(5):421-433, 1997.

[2] H. Abrach, S. Bhatti, J. Carlson, H. Dai, J. Rose, A. Sheth, B. Shucker, J. Deng, and R. Han. Mantis: System support for multimodal networks of in-situ sensors. In 2nd ACM International Workshop on Wireless Sensor Networks and Applications (WSNA), pages 50-59, 2003.

[3] The Arena Project. http://www.cdt.luth.se/projects/arena/, June 2004.

[4] Bluetooth Module WML-C10 Class 2. MITSUMI ELECTRIC CO., LTD. http://www.mitsumi.com, June 2004.

[5] Bluetooth Special Interest Group. Bluetooth Core, Specification of the Bluetooth System, Version 1.1. February 2001. https://www.bluetooth.org/spec/, June 2004.

[6] S. Boll and U. Westermann. Mediether: an event space for context-aware multimedia experiences. In Proceedings of the 2003 ACM SIGMM workshop on Experiential telepresence, pages 21-30. ACM Press, 2003.

[7] K. Cheverst, N. Davies, K. Mitchell, and A. Friday. Experiences of developing and deploying a context-aware tourist guide: the GUIDE project. In Proceedings of the 6th annual international conference on Mobile computing and networking (MOBICOM 2000), pages 20-31. ACM Press, 2000.

[8] P. De Bra, P. Brusilovsky, and R. Conejo. Tv scout: Lowering the barrier to personalized tv program recommendation. In Proceedings of the 2nd International Conference on Adaptive Hypermedia and Adaptive Web Based Systems (AH 2002), pages 58-68. Springer-Verlag, 2002.

[9] A. K. Dey, D. Salber, and G. D.Abowd. A conceptual framework and a toolkit for supporting the rapid prototyping of context-aware applications. Anchor article of a special issue 
on context-aware computing in the Human-Computer Interaction (HCI) Journal, 16:97-166, 2001.

[10] Dunkels. lwIP - a lightweight TCP/IP stack. http://www.sics.se/ adam/lwip/, November 2003.

[11] H. W. Gellersen, A. Schmidt, and M. Beigl. Multi-sensor context-awareness in mobile devices and smart artifacts. Mobile Networks and Applications, 7(5):341-351, 2002.

[12] J. Hill and D. Culler. A wireless embedded sensor architecture for system-level optimization. Technical report, U.C. Berkeley, 2001.

[13] J. Hill, R. Szewczyk, A. Woo, S. Hollar, D. Culler, and K. Pister. System architecture directions for network sensors. In Proceedings of the ninth International Conference on Architectural Support for Programming Languages and Operating Systems, pages 93-104, 2000.

[14] X. Jiang and J. Landay. Modeling privacy control in contextaware systems. IEEE Pervasive computing, 1(3):59-63, July-Sept 2002.

[15] M. Lamming and M. Flynn. Forget-me-not: intimate computing in support of human memory. In Proceedings FRIEND21 Symposium on Next Generation Human Interfaces, 1994.

[16] Lassen SQ GPS. Trimble Navigation Limited. http://www.trimble.com/lassensq.html, June 2004.

[17] lwBT - a lightweight Bluetooth stack. http://www.sm.luth.se/ conny/lwBT/, November 2003.

[18] Microcontroller M16C/62M. Renesas Technology Corporation. http://www.renesas.com/eng/, June 2004.

[19] R. Min, M. Bhardwaj, S. Cho, A. Sinha, E. Shih, A. Wang, and A. P. Chandrakasan. An architecture for a power-aware distributed microsensor node. In IEEE Workshop on Signal Processing Systems (SiPS '00), pages 581-590, 2000.

[20] J. Nord, K. Synnes, and P. Parnes. An Architecture for Location Aware Applications. In Proceedings of Proceedings of Hawaii International Conference on System Sciences (HICSS-35), january 2002. Nominated to Best Paper.

[21] D. Olsson and A. Nilsson. Mep: a media event platform. Mobile Networks and Applications, 7(3):235-244, 2002.

[22] G. S. Pingali, A. Opalach, Y. D. Jean, and I. B. Carlbom. Instantly indexed multimedia databases of real world events. IEEE Transactions on Multimedia, 4(2):269-282, 2002.

[23] Polar T61 coded transmitter. Polar Electro Oy. http://www.polar.fi/polar/index.html, June 2004.

[24] A. Ranganathan and R. H. Campbell. An infrastructure for context-awareness based on first order logic. Personal and Ubiquitous Computing, 7(6):353-364, 2003.

[25] B. J. Rhodes. The wearable remembrance agent: a system for augmented memory. In The Proceedings of The First International Symposium on Wearable Computers (ISWC '97), pages 123-128. IEEE Computer Society Press, October 1997.

[26] M. Román, C. K. Hess, R. Cerqueira, A. Ranganathan, R. H. Campbell, and K. Nahrstedt. Gaia: A middleware infrastructure to enable active spaces. IEEE Pervasive Computing, 1(4):74-83, Oct-Dec 2002.

[27] Survey; questions and answers. http://www.sm.luth.se/ qwazi/vasaloppet/, June 2004.
[28] K. Synnes, J. Nord, and P. Parnes. Location Privacy in the Alipes platform. In Proceedings of Hawaii International Conference on System Sciences (HICSS-36), january 2003.

[29] Testbed Botnia. http://testplats.com/doc/aboutbotnia/se, June 2004.

[30] Vasaloppet. www.vasaloppet.se, June 2004.

[31] S. Voida, E. D. Mynatt, B. MacIntyre, and G. M. Corso. Integrating the virtual and physical context to support knowledge workers. IEEE Pervasive Computing, 1(3):73-79, July - September 2002.

[32] V.Raghunathan, C. Schurgers, S. Park, and M. B. Srivastava. Energy-aware wireless microsensor networks. IEEE Signal Processing Magazine, 19(2):40-50, 2002.

[33] R. Want, B. N. Schilit, N. I. Adams, R. Gold, K. Petersen, D. Goldberg, J. R. Ellis, and M. Weiser. An overview of the parctab ubiquitous computing experiment. IEEE Personal Communications, 2(6):28-43, December 1995.

[34] E. Welsh, W. Fish, and J. Frantz. Gnomes: A testbed for low-power heterogeneous wireless sensor networks. In Proceedings of the 2003 International Symposium on Circuits and Systems, pages 836-839, 2003.

[35] H. Wu. Sensor Data Fusion for Context-Aware Computing Using Dempster-Shafer Theory. PhD thesis, Carnegie Mellon University, Pittsburgh, Pennsylvania 15213, December 2003.

[36] Å. Östmark, C. Öhult, J. Eriksson, P. Lindgren, and J. Delsing. A wireless network of EIS devices. In Proceedings of the 21th IEEE Instrumentation and Measurements Technologhy Conference, 2004. 